\section{Prediction of postoperative urinary tract infection in men undergoing cardiac surgery by preoperative measurement of urine flow}

A survey of septicaemia after open heart surgery in the Northern regional cardiothoracic surgical centre showed that $30 \%$ of cases of such septicaemia originated in the urinary tract ( $R$ Freeman, F K Gould, M R Jones, unpublished observation). Of 14 affected patients, only one had a detectable abnormality on examination of a preoperative midstream urine specimen. Some candidates for open heart surgery might have asymptomatic bladder dysfunction with outflow tract obstruction and a residual urine volume and might then develop postoperative urinary tract infection. Measurement of urine flow by a simple flowmeter is a well established technique in urology and provides a good guide to the presence of outflow tract obstruction in men. ${ }^{12}$ We measured preoperative urine flow in men about to undergo cardiac surgery to screen for asymptomatic outflow tract obstruction. We also evaluated the predictive value of symptomatic inquiry and the conventional preoperative midstream urine specimen.

\section{Patients, methods, and results}

We studied all men admitted for open heart surgery from March to June 1983 (61 patients ages $23-75$ (mean $51 \cdot 7$ ) years). On admission every patient was interviewed and gave a full urological history; gave a midstream urine sample for microscopy and culture; and visited the urology outpatient department for measurement of peak urine flow.

The surgical team did not know any details of the assessment apart from the result of the examination of the preoperative midstream urine sample, and the care of the patient was standard for the unit. In particular, all patients underwent urethral catheterisation; the catheter was usually removed the day after operation. A midstream urine sample was obtained on the patient's return to the convalescent ward and if possible on at least one further occasion before discharge. All urine samples were cultured, and those yielding a pure growth of over 100000 colony forming units $/ \mathrm{ml}$ were taken as diagnostic of urinary tract infection. Finally, a senior member of the urology department who had not taken part in the study classified the results of the flow measurements into three categories (normal, abnormal, or invalid owing to technical problems).

No abnormalities were seen in preoperative midstream urine samples on microscopy or culture, but six patients $(9 \cdot 8 \%$ ) acquired postoperative urinary tract infection. None of the patients progressed to septicaemia. The table shows that preoperative symptoms or a known history of urological disease, or both, were not associated with postoperative urinary tract infection but that patients who had an abnormal urine flow rate before operation were significantly more likely to develop postoperative urinary tract infection. Patients throughout the age range tested had abnormal flow rates, the youngest such patient being 36 .

Relations between preoperative flow rate, history of urological disease, recent urinary tract symptoms, and development of postoperative urine infection

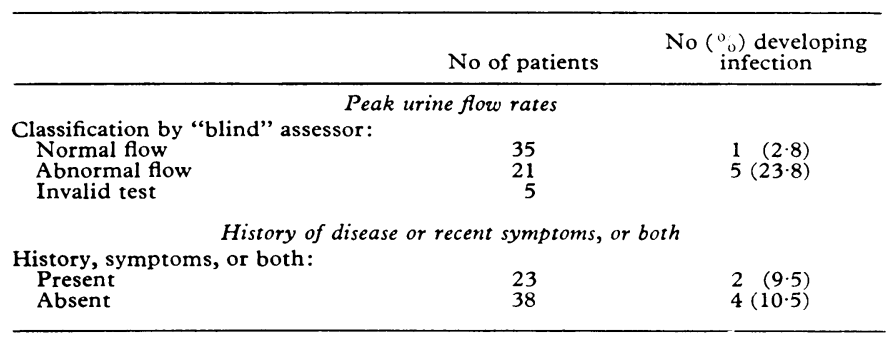
Differences in incidences of infection between: patients with normal and abnormal
flow rates, $p<0.5$; patients with and without a history or recent symptoms, or both, NS.

\section{Comment}

We showed that an abnormally low urine flow rate in men about to undergo open heart surgery defines a group of patients in whom urinary tract infection is more likely to occur $\left(24^{\circ}{ }_{0} v 3{ }_{0}^{\circ}\right)$; a history of urological disease or recent symptoms did not predict the likelihood of infection.

Ideally urine flow should be measured on more than one occasion and the possibility of residual urine volume excluded by ultrasound examination. Neither of these was possible in this study, although further tests would probably have permitted proper classification of the five patients in whom the urine flow measurement was invalid.

We found that almost $10 \%$ of men undergoing cardiac surgery developed postoperative urinary tract infection. Other groups of men undergoing surgery that entails urethral catheterisation and restricted mobility - for instance, hip replacement or some forms of abdominal surgery-might have a similar incidence of outflow tract obstruction. The finding that patients at risk can be predicted by measurement of the preoperative urine flow rate should permit rational preventive measures.

We thank the nursing staff of the urology department and cardiothoracic surgical unit at Freeman Hospital for their cooperation and help, and the cardiac surgeons for permission to study their patients. We also thank Mr D M Essenhigh for the original suggestion and for his encouragement.

${ }^{1}$ Drach GW, Layton TN, Binard WJ. Male peak urinary flow rate: relationships to volume voided and age. $\mathcal{F}$ Urol 1979;122:210-4.

${ }^{2}$ Shoukry ISJG, Elhilali MM, Dutarte D. Role of uroflowmetry in the assessment of lower urinary tract obstruction in adult males. $\mathrm{Br} \mathcal{F}$ Urol 1975;47:559-66.

(Accepted 3 October 1983)

Department of Microbiology, Freeman Hospital, Newcastle upon Tyne NE7 7DN

F K GOULD, MB, BS, registrar

R FREEMAN, MB, CHB, MRCPATH, consultant

Correspondence to: Dr R Freeman.

\section{Guide wire manipulation of Crosby jejunal biopsy capsule under fluoroscopic control}

The reported prevalence of coeliac disease is one case per 1850 population in the United Kingdom, ${ }^{1}$ and in certain areas of western Ireland it is as high as one per $300 .^{2}$ The number of patients with the disease known to the Coeliac Society is said to be far less than the national prevalence. ${ }^{3}$ Diagnosis depends on the appearances of a jejunal biopsy specimen, which is obtained most commonly with a Crosby capsule, which is swallowed by the patient, passed into the jejunum, fired, and retrieved. Placing the capsule in the jejunum may take two hours or more. If this time could be reduced more patients with minor symptoms might be recommended for biopsy. ${ }^{4}$

\section{Technique}

The adult Crosby capsule (Ferraris, Edmonton, London) is used with Portex P3325 tubing, which has a suitable degree of stiffness and appropriate internal diameter. Teflon coated Cordis guide wire (type 501250 ) is used rather than a Seldinger wire. ${ }^{5}$ It is $150 \mathrm{~cm}$ long and has a diameter of $9 \cdot 7$ $\mathrm{mm}$. It may be reused after soaking in glutaraldehyde solution.

As soon as the patient has swallowed the capsule fluoroscopy is carried out with the screening table in the erect position, so that the capsule is located and the extent of any coiling determined. The floppy end of the guide wire is then inserted into the tubing, and while the position of the wire is maintained the tube is pulled back over it until any coils are unlooped. The principle of the operation is in the interplay of the tube and guide wire. The guide wire stiffens the tube, thus helping it to pass without coiling. Partial withdrawal of the guide wire enables the capsule end of the tube to be passed easily around curves, when the operator may advance the tube and wire together.

With the antrum and pylorus outlined by air in the stomach the length of the guide wire within the tube is adjusted, its tip determining the point from which the pliable end of the tube will curve against the stomach wall. The patient's position can be adjusted to allow gravity to help direct the capsule. Once the capsule faces the pylorus the guide wire is fully inserted into the tubing and together they are moved gently forward through the pylorus. The guide wire is then withdrawn to a point just inside the antrum and the forward movement is repeated. Once the capsule is within the second part of the duodenum the guide wire can safely be advanced over the superior flexure, thus enabling the capsule (with a judged amount of tubing free of wire) to pass around the remainder of the duodenum. In the same way the capsule should pass easily around the duodenojejunal flexure. If it becomes lodged in the duodenum the patient should be asked to cough or the area should be palpated with a (lead gloved) hand.

Duration of manipulation- The table shows that the time taken to move the capsule from the stomach to a point about $5 \mathrm{~cm}$ past the duodenojejunal 
Duration and results of manipulation in 20 consecutive patients

\begin{tabular}{cccl}
\hline Case No & $\begin{array}{c}\text { Manipulation time } \\
\text { (minutes) }\end{array}$ & $\begin{array}{c}\text { Screening time } \\
\text { (minutes) }\end{array}$ & \multicolumn{1}{c}{ Result } \\
\hline 1 & $6 \cdot 5$ & $4 \cdot 5$ & Normal \\
2 & $4 \cdot 5$ & $3 \cdot 0$ & Coeliac disease \\
3 & $4 \cdot 75$ & $3 \cdot 5$ & Normal \\
4 & $4 \cdot 5$ & $2 \cdot 5$ & Coeliac disease \\
5 & $9 \cdot 0$ & $4 \cdot 25$ & Normal \\
6 & $3 \cdot 5$ & $2 \cdot 25$ & Normal \\
7 & $8 \cdot 5$ & $5 \cdot 25$ & Normal \\
8 & $12 \cdot 0$ & $4 \cdot 75$ & Normal \\
9 & $9 \cdot 75$ & $4 \cdot 5$ & Normal \\
10 & $8 \cdot 0$ & $4 \cdot 25$ & Normal \\
11 & $1 \cdot 5$ & $0 \cdot 75$ & Coeliac disease \\
12 & $7 \cdot 5$ & $3 \cdot 75$ & Normal \\
13 & $14 \cdot 0$ & $6 \cdot 5$ & Normal \\
14 & $13 \cdot 0$ & $6 \cdot 0$ & Normal \\
15 & $3 \cdot 5$ & $2 \cdot 0$ & Normal \\
16 & $7 \cdot 5$ & $4 \cdot 75$ & Coeliac disease \\
17 & $4 \cdot 75$ & $2 \cdot 5$ & $5 \cdot 0$ \\
18 & $7 \cdot 0$ & $5 \cdot 0$ & \\
19 & $7 \cdot 5$ & $2 \cdot 75$ & \\
20 & $4 \cdot 0$ & & \\
\hline
\end{tabular}

*Capsule failed to pass through pylorus because stomach was axially rotated.

flexure in 20 cases varied between 1.5 and 14 (mean 7.4 ) minutes. The average screening time was four minutes. In only one patient (case 10) had the capsule already passed through the pylorus when he was first screened in the $x$ ray department. In all other patients the capsule was near the pylorus when they were first screened.

\section{Comment}

Fluoroscopy, though important to the procedure, is used intermittently and kept to a minimum. The technique is rapid, simple, and much less uncomfortable for the patient than other methods of placing capsules.

I thank Dr R F Harvey (consultant) and Dr T Gill, department of gastroenterology, and Dr A J Longstaff (consultant), department of radiology, Frenchay Hospital, Bristol, for their help and constructive criticism.

' McCrae WM. Inheritance of coeliac disease. 7 Med Genet 1969;6:129-31.

"2 Mylotte M, Elan-Mitchell B, McCarthy CF, et al. Incidence of coeliac disease in the West of Ireland. Br Med F 1973; : 703-5.

${ }^{3}$ Swinson CM, Levi AJ. Is coeliac disease under diagnosed? $\mathrm{Br} \mathrm{Med} \mathrm{F}$ $1980 ; 281: 1258-60$.

${ }^{4}$ Logan REA, Tucker G, Rifkind EA, et al. Changes in the clinical features of coeliac disease in adults in Edinburgh and the Lothians. $\mathrm{Br} \mathrm{Med} \mathcal{F}$ $1983 ; 286: 95-7$

${ }^{5}$ Fric P, Lepsik J. Use of Ödman-Ledin catheter and Seldinger wire with Crosby capsule. Gut 1965;16:101.

(Accepted 3 October 1983)

General X Ray Department, Frenchay Hospital, Bristol BS16 1LE ROBERT L LAW, DCR, superintendent radiographer

\section{Self poisoning with enalapril}

Enalapril (MK-421; $N$-(1-ethoxycarbonyl-3-phenylpropyl)-L-alanylL-proline hydrogen maleate) is an angiotensin converting enzyme inhibitor $^{1}$ that is useful in managing patients with hypertension and congestive heart failure refractory to conventional treatment. ${ }^{23}$ It is a prodrug that must be hydrolysed in vivo to its parent diacid compound enalaprilic acid (MK-422), which is biologically effective. Long term blockade of the renin angiotensin system with this agent is usually well tolerated, but no information has been available on the effects of acute overdosage. We report on a patient who tried to commit suicide by ingesting a large dose of both enalapril and oxazepam, a benzodiazepine.

\section{Case report}

The patient was a 46 year old woman with severe essential hypertension and a history of right side cerebrovascular accident. Her blood pressure had been well controlled with enalapril ( $20 \mathrm{mg}$ twice daily) and chlorthalidone (100 mg once daily) for two years when she attempted to commit suicide by ingesting $300 \mathrm{mg}$ enalapril and $225 \mathrm{mg}$ oxazepam. On admission four hours later she was stuporous but had not developed respiratory distress.
Blood pressure was $100 / 60 \mathrm{~mm} \mathrm{Hg}$ and heart rate 80 beats/minute. During the subsequent hours blood pressure oscillated around $80-100 / 50-80 \mathrm{~mm} \mathrm{Hg}$ and consciousness progressively improved. Occasional ventricular premature contractions were recorded, but her pulse rate showed no tendency to accelerate. After infusion of $2000 \mathrm{ml}$ sodium chloride $0.9 \%$ and $250 \mathrm{ml}$ plasma intravenous blood pressure rose to $110-125 / 70-85 \mathrm{~mm} \mathrm{Hg}$, within the first 24 hours after admission.

She was discharged after three weeks' uneventful observation; her blood pressure still had not reached hypertensive values, though no antihypertensive treatment had been given. Ten days later enalapril and chlorthalidone had to be restarted. Laboratory profiles remained normal throughout the month after drug overdosage.

The figure shows the time course of the changes in the serum concentration of enalaprilic acid, plasma angiotensin II concentration, and plasma angiotensin converting enzyme activity after the attempted suicide. Plasma angiotensin II and serum enalaprilic acid concentrations were measured by radioimmunoassay and plasma angiotensin converting enzyme activity by a radioenzymatic method. ${ }^{34}$ Ten hours after ingestion of $300 \mathrm{mg}$ enalapril the serum concentration of enalaprilic acid had reached $1450 \mu \mathrm{g} / \mathrm{l}$ and plasma angiotensin converting enzyme activity had been completely suppressed. At the same time the serum concentration of enalapril was $50 \mu \mathrm{g} / \mathrm{l}$. This was estimated as the difference between concentrations of enalaprilic acid before and after in vitro hydrolysis of the residual enalapril contained in the sample tested. Only from her fourth day in hospital did the plasma angiotensin concentration start to increase and plasma angiotensin converting enzyme to resume its activity. Blood pressure remained normal despite the unblocked renin angiotensin system.
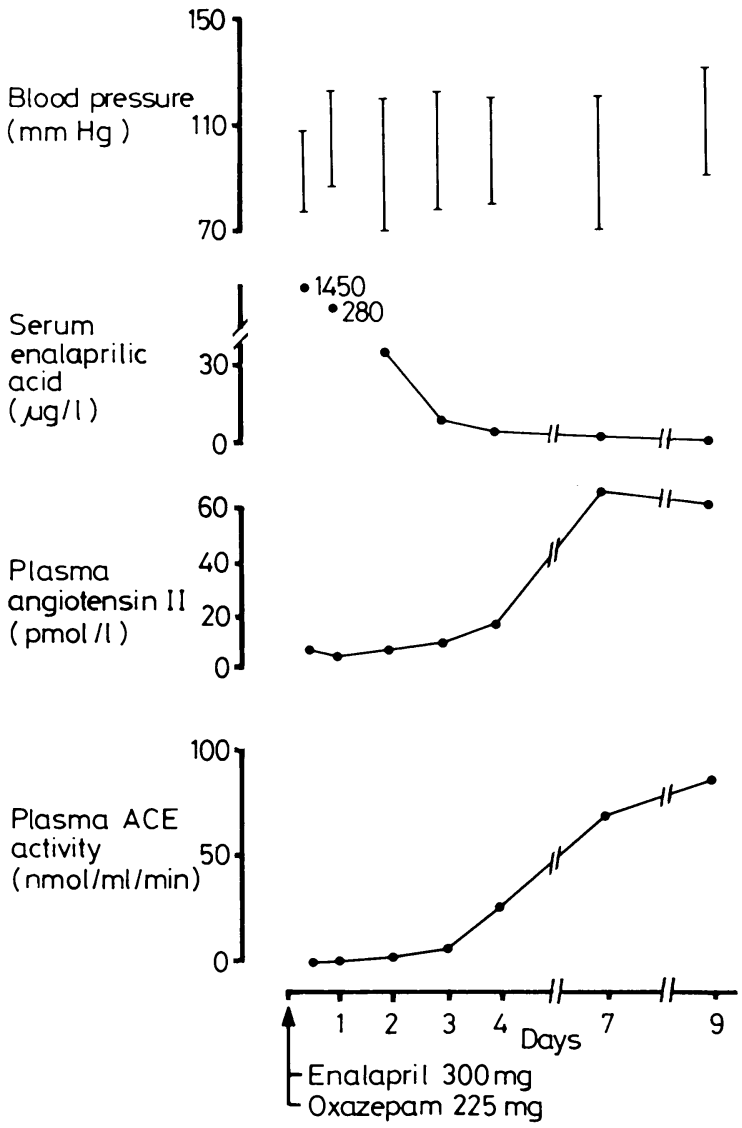

Changes in blood pressure, serum concentration of enalaprilic acid, plasma angiotensin II concentration, and plasma angiotensin converting enzyme (ACE) activity after ingestion of $300 \mathrm{mg}$ enalapril and $225 \mathrm{mg}$ oxazepam.

Conversion: SI to traditional units-Plasma angiotensin II: $1 \mathrm{pmol} / \mathrm{l} \approx$ $1 \mathrm{pg} / \mathrm{ml}$.

\section{Comment}

Enalapril is a potent long acting angiotensin converting enzyme inhibitor that has to be hydrolysed in vivo to its active metabolite enalaprilic acid. In our patient serum of enalaprilic acid concentrations 10 hours after ingestion of $300 \mathrm{mg}$ enalapril were over 100 times higher than necessary to inhibit angiotensin converting enzyme. ${ }^{4}$ The capacity to hydrolyse enalapril was not exceeded because at the same time there was little intact drug in the serum.

Overdosage with enalapril and oxazepam did not cause any serious complications. The hypotensive episode shortly after overdosage was well tolerated and easily reversed by supportive infusion of fluid. 